\title{
Parallel Computing With R: A Brief Review
}

\begin{abstract}
Dirk Eddelbuettel $\left.\right|^{1}$
${ }^{1}$ University of Illinois, Urbana-Champaign, IL, USA

This version was compiled on April 7, 2020

Parallel computing has established itself as another standard method for applied research and data analysis. The $\mathbf{R}$ system, being internally constrained to mostly singly-threaded operations, can nevertheless be used along with different parallel computing approaches. This brief review covers OpenMP and Intel TBB at the CPU- and compiler level, moves to process-parallel approaches before discussing message-passing parallelism and big data technologies for parallel processing such as Spark, Docker and Kubernetes before concluding with a focus on the future package integrating many of these approaches.
\end{abstract}

OpenMP, OpenMPI, Kubernetes, Spark, Parallel Computing, R

\section{Introduction}

A decade ago, Schmidberger et al. (2009) surveyed parallel computing with the R language and environment. Their overview article provided a useful road map for several years to come, and aided both researchers and applied statisticians in making sense of a rapidly changing landscape.

Roughly a decade later, a lot has changed. Entirely new technologies such as Docker have emerged (see Boettiger and Eddelbuettel (2017) for a number of use cases in the context of R). Cloud computing, which was just beginning to make its case when the earlier article was written, is now a dominant use case. Standard high-performance computing, a focus of the earlier article, is still around but somewhat overshadowed by cloud computing. New and more commercially-focused integrations such as Hadoop and Spark offer a different take on data parallelism and have become standard application in industry in part by their ability to cope with 'big(ger) data' requirements. At the same time, deep learning emerged as a new field and brought with it several new new computing frameworks. While a variety of competing frameworks are available, TensorFlow (Abadi et al., 2015) and PyTorch (Paszke et al., 2019) can be considered as the two most prominent examples.

At the other end of the hardware spectrum, processing units offer more and more parallelism directly at the center of the operations. Central processing units (CPUs), still primarily provided by Intel and AMD, now offer several dozen cores permitting both multi-threaded and multi-process applications. Graphical processing units (GPUs) continue to play a very important role as well by offering hundreds of cores (albeit at a lower clock speed). Many high-end super-computing installations (as e.g. evidenced by the TOP500 listings) combine CPUs and GPUs. A whole new entrant in the hardware space are dedicated components for deep learning which are often data center-vendor specific. One example is called Tensor Processing Units (TPUs) and offered by Google, the company behind TensorFlow.

During this time, some parts remained the same. Compiler-supported "local" parallelism such as OpenMP has become fairly standard. It is (within limits) used by $\mathrm{R}$ itself as well as in a number of application packages as we will discuss in more detail below. But some new entrants emerged as well. The future package by Bengtsson (2019a) offers an elegant unified abstraction over both "local" and "remote" (i.e., distributed) variants, and has become popular and widely adopted.

This paper updates the road map by Schmidberger et al. (2009) by surveying several of these more recent entries. We begin by defining a number of terms to facilitate the discussion. We then start from the inside out, focusing first on OpenMP and related compiler-driven technologies that are very 'local' to the core of the execution. Next, we consider more conventional high-performance and parallel computing approaches. We then turn to a few of the newer frameworks which have arisen in the last decade, and which can also play an important role in parallel computing. Thereafter, we discuss the 
futures package as the key application for $\mathrm{R}$ in this space. A brief summary concludes.

\section{Definitions}

Before discussing actual technologies and approaches for parallel computing with $\mathrm{R}$, it is helpful to review and clarify a number of terms used in the discussion. This section recalls and illustrates a number of standard definitions.

Sequential Execution. R as a language and environment is reasonably well established and understood. A combination of dynamic typing, lazy functional evaluation and object-orientation (in several flavors) makes for a somewhat unique combination as discussed by Morandat et al. (2012). One side-effect of this design is that core operations are undertaken in single-threaded mode, or, in other words, sequentially. Sequential computing consists of operations executed in strict sequence, and following a predetermined ordering. It may be the simplest unit of computation (beyond a single instruction): a subroutine or function in which each operation happens strictly after the preceding one.

As an example and to fix the discussion on some concrete illustrations, consider the following (simplified) code example. We omit error and argument checking for the sake of brevity.

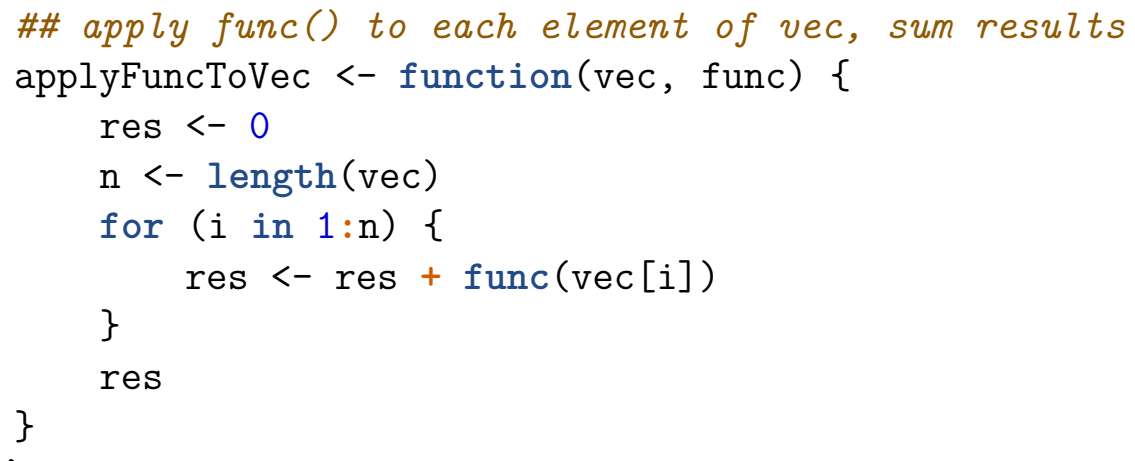

The principal computational cost of the function is likely the repeated evaluation of the supplied function func() (provided its complexity is sufficiently different from an "empty" function in which case the overhead of the loop, and the repeated function calls dominate). As should be clear from the code, all these operations are executed sequentially with each of the $n$ calls occurring after the other.

Such a sequential setup provides the baseline against which parallel execution can be measured in terms of both benefits (such as lower execution time) and costs (such as increased memory or communication overhead).

Concurrent Execution. The first generalization from sequential execution is concurrent execution. Concurrency is commonly defined as running more than one task in non-overlapping time periods. In other words, at any one time only one task is executed however processing switches (possibly frequently) between multiple tasks. A classic example is a computer operating system advancing multiple tasks on a single CPU and single core system: tasks are executed in turns, and possibly in small increments. This can give the illusion of several things happening at once; yet in reality this really is merely efficiently switching between tasks.

Hence, a key aspects of concurrency is the task-switching cost which, at a minimum, involves memory access and moves to bring the different tasks to the CPUs. 
Parallel Execution. The polar opposite to sequential execution is parallel execution. It is defined as multiple operations happening in overlapping time periods. This requires multiple execution units: maybe cores within a CPU, maybe multiple CPUs (possibly each with multiple cores), and maybe multiple computers systems.

An important distinction hinges on the actual task being executed in parallel, and its properties. A key class of problems are known as data parallel problems. Each sub-task $i$ is independent of each other sub-task $j$. In such a case, no constraints are imposed on the task scheduling. In fact, linear speedups would be possible were it not for the actual overhead in scheduling and coordinating between the parallel workloads being executed. A classic example is our simple function call above when the supplied function func () depends only on the current vector element. Now multiple chunks of the vector can be operated on in parallel, and the final result can be aggregated. (Without going into finer details, this is the core principle behind 'map-reduce' computing approaches.)

Of course, the opposite also holds: should the tasks have data dependence, then parallel (or even concurrent) execution is much more challenging. A simple example would be an instance of func() where the $i$-th value depends on the preceding value with index $i-1$.

Single Instruction Multiple Data (SIMD). Another class of CPU and compiler-centric parallel instructions is Single Instruction Multiple Data, or SIMD. A good overview and history is offered by Wikipedia Contributors (2019c).

The history of SIMD goes back to when 'vector computers' were the original supercomputers. However, such instruction sets are now also common in high-end workstations and servers. The current top-of-the line offering is AVX-512 instruction sets which operate on 512 bits at a time. With standard double-precision floating point variables taking eight bytes, or 64 bits, we see eight of these being addressable via AVX-512 instruction sets. It should be noted that only higher-end server CPUs and chip sets currently offer this. For example, the author's workstation with a more modest Intel Core i7 processor, albeit at six cores, does not offer it.

As such techniques are very dependent on the actual hardware used, and vary with the hardware, benchmarking toolkits are important as they can help in comparing across setups, and in calibration. One example of such an (open source) toolkit is Likwid (Treibig et al., 2010; Roehl et al., 2014).

\section{Local Parallelism via Shared Memory Architecture}

Parallel computing that is accessible from $\mathrm{R}$ can be implemented via compiled extensions to $\mathrm{R}$ by relying on compiler-specific extensions. This section discusses two: OpenMP, an industry standard for shared-memory parallelism, and Intel Thread Building Blocks, which is similar to OpenMP but offers a higher abstraction level.

OpenMP. A key technology for parallel execution of compiled code is provided by OpenMP which was introduced by Dagum and Menon (1998). It has become an industry standard with the current version being OpenMP 5.0 published in November 2018. Support for OpenMP is generally provided by the compiler. For the GNU Compiler Collection, all of gcc, g++ and gfortran can use OpenMP directives. $\mathrm{R}$ itself has had support on all platforms since release 3.4.0 in April 2017.

As mentioned earlier, and because of the dynamic nature of $\mathrm{R}$, care has to be taken in order to use OpenMP as R itself is single-threaded. That said, OpenMP has long been used by R itself (conditional of course on the particular build and architecture supporting it). The use of OpenMP ranges from an option for matrix multiplication still labeled "experimental" (see the "matprod' entry in help (options)) to possible OpenMP/SIMD enhanced checks for non-finite values as well as parallel column sums (all 
in src/main/array.c) and to the use in the implementation of the $\operatorname{dist}($ ) function in the base $\mathrm{R}$ package stats.

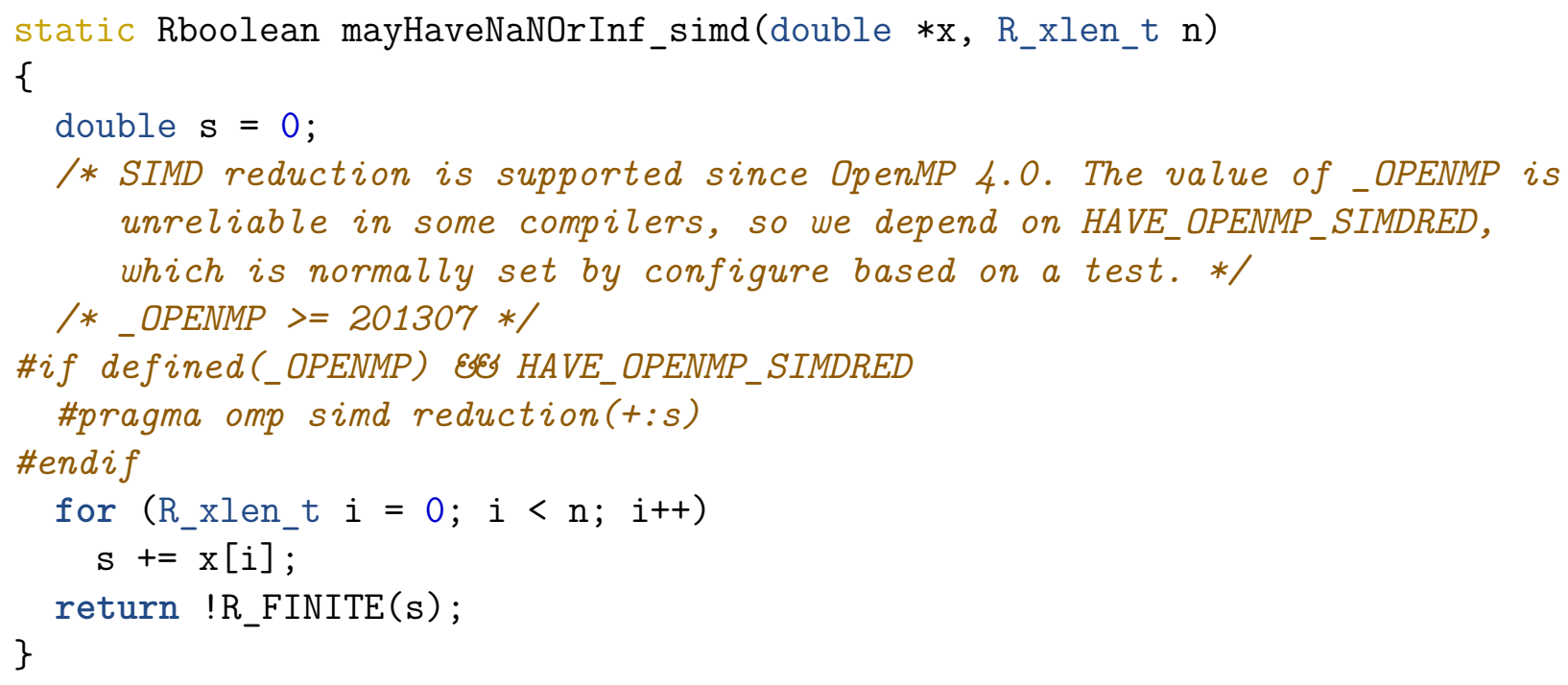

This is a typical usage pattern for OpenMP. The C code is ornamented with a \#pragma which is itself conditional on OpenMP as well as SIMD reductions with OpenMP being available. If so the compiler will generate more efficient parallel code: in lieu of the basic loop touching each vector element in sequence, SIMD operations can do so with some parallelism.

As noted above, SIMD support is also dependent on the particular CPU model. So for a concrete example (which is shown below), we compare a simpler OpenMP parallel for loop with a normal, non-parallelized loop. It can be built via Rcpp: : sourceCpp ("filename.cpp"), and relies on the Rcpp (Eddelbuettel et al., 2019a; Eddelbuettel and Balamuta, 2018) and microbenchmark (Mersmann, 2019) packages. The benchmarking section at its end will run when the code is sourced.

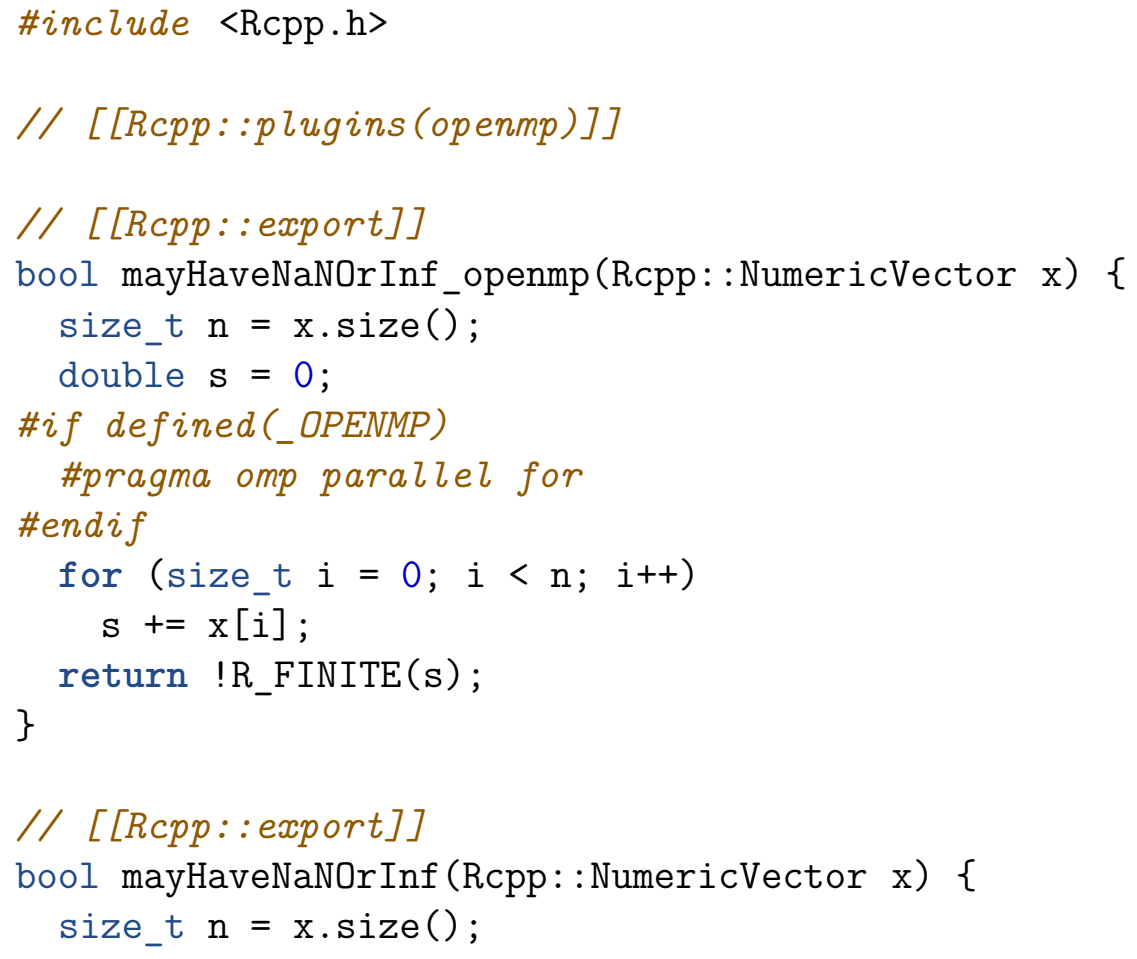




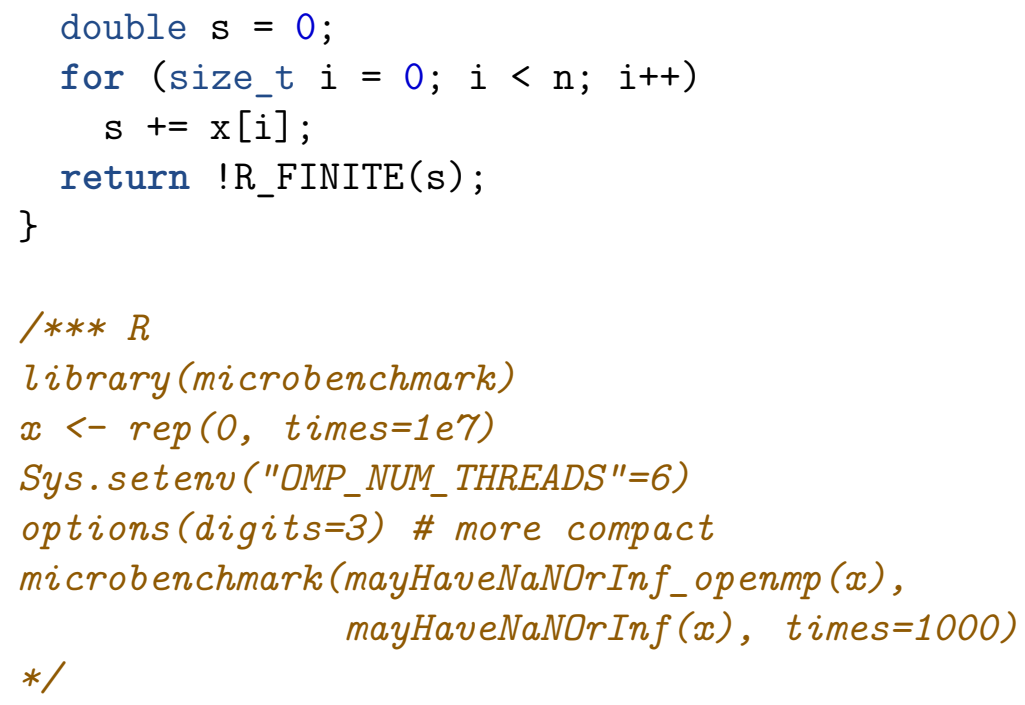

On an Intel Core i7-8700 with six cores, we obtain a roughly four-fold improvement (when measuring median time) between the conventional loop, and the OpenMP-parallelized loop. That is a significant speed gain for an operation that may be executed quite frequently, and shows why OpenMP is a compelling tool. However, it also shows that OpenMP requires some familiarity with programming at the source level (in C, C++, or Fortran).

OpenMP is a key technology for parallel computing with R. How to enable it is described in some detail in Section "OpenMP support" of the "Writing R Extensions" manual (R Core Team, 2019). We should also note that different operating system vary somewhat in how well they support OpenMP. Since the R 3.4.0 release in 2017, it is generally available across the platforms supported by R. The rich corpus of package source code presented by the CRAN repositories offers some insights in how prevalent the usage of OpenMP among $\mathrm{R}$ packages is. We find 211 packages using OpenMP via the $\mathrm{R}$ Core team recommended use of common make variables set by $\mathrm{R}$ during its build. Here, SHLIB_OPENMP_CFLAGS is the C compiler flags, similar flags exist for C++ as well as for Fortran. Running a simple grep among the Makevars $\{$, . in\} files reveals 355 packages using this instruction. This can be seen as an upper bound as some packages recommend use of the build variable in order to benefit from OpenMP use in the code they provide for other packages. An example of this practice is RcppArmadillo (Eddelbuettel et al., 2019b)) offering parallel linear algebra and utility code for use by other packages.

Going one step further, we can look for actual \#pragma omp code in the package sources. We consider code in or below the src/ directory, and also in or below the inst/include directory from where it may be accessible to other packages via header includes. We find that over two-hundred $\mathrm{R}$ packages on CRAN use OpenMP. Among these, StanHeaders (Goodrich et al., 2019) leads with 246 instances, the RViennaCL package (Determan Jr., 2019) has 232, spMC (Sartore, 2019) is not far behind with 226 and packages data.table (Dowle and Srinivasan, 2019), xgboost (Chen et al., 2019), and RNiftyReg (Clayden et al., 2019) each have over 90. This informal inspection of the CRAN package sources shows that OpenMP is indeed widely-used among R packages.

Intel Thread Building Blocks (TBB). A closely-related technology is the Intel Thread Building Blocks (or Intel TBB for short). Like OpenMP, it is a means to have the compiler generate parallel code but in this case at a somewhat higher abstraction level, and only via $\mathrm{C}++$.

The RcppParallel package (Allaire et al., 2019) wraps the Intel TBB making it straightforward to deploy this technology, especially if $\mathrm{R}$ and $\mathrm{C}++$ bindings are already being used. The package comes 
with documentation and examples showing typical Map-Reduce patterns where the Intel TBB can automatically determine the optimal 'chunk' sizes when deciding how to parallelise, say, operations on a vector just as in our first example above.

The following code example, taken from the RcppParallel documentation, illustrates how a worker object for a 'summation' can be set up. The example is similar to our initial example as it operates element-wise on a vector. Here, a key element is in the operator () implementation: by giving begin and end indices, we allow the worker to operator on the contiguous chunk of the vector defined by this index range. One level up, TBB deals with appropriate 'chunking' and submits each 'chunk' to the worker shown here. A join() operation updates an incoming Sum variable by adding the results of the computations on just this chunk.

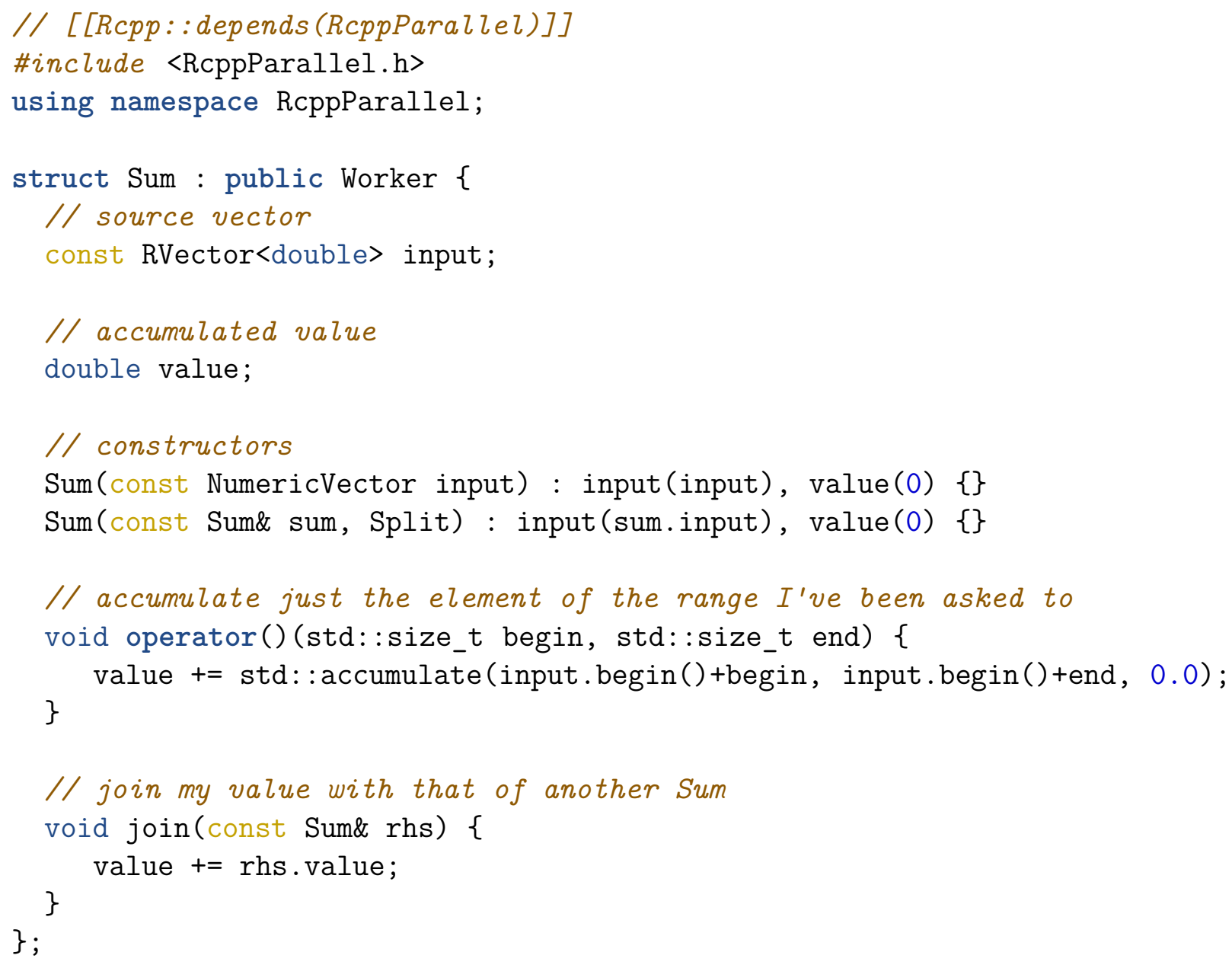

The attractive high-level abstraction of Intel TBB is clearly apparent in the following example. We combine a Sum instance (utilizing the Worker shown in the previous example) with a given numeric vector $\mathrm{x}$, and pass it to parallelReduce () along with the dimensions (here the entire vector length). Intel TBB takes care of appropriate task and chunk size as well as scheduling, even offering what is referred to as 'work-stealing' where idle cores can take up processing from other queues when their own queue is empty. But these details are hidden by the implementation-we simple return the computed (scalar) value at the end. 


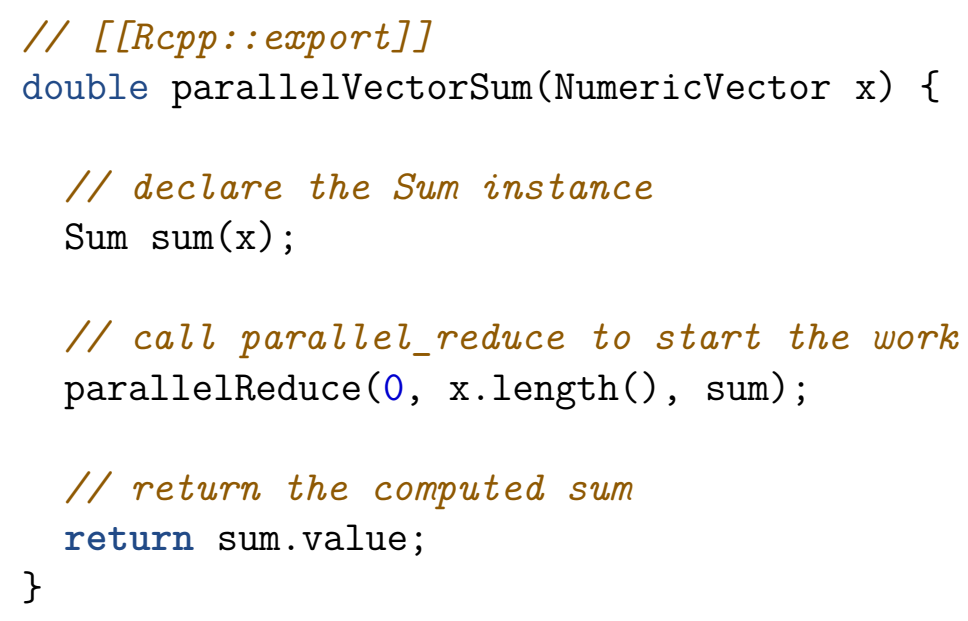

Intel TBB is an attractive solution if the $\mathrm{C}++$ language can be used. It is also not restricted to hardware manufactured by Intel as AMD CPUs work as well. For R users, the RcppParallel package (Allaire et al., 2019) makes it accessible from $\mathrm{R}$ by supplying all required components, notably the Intel TBB header library itself, in the convenient form of a package.

Other Related Aspects. One important topic related to both OpenMP and Intel TBB is the use of random number generators (RNGs). For sequential code, an RNG implementation can remain stateful and deliver high-quality random draws. For a user of $\mathrm{R}$, choosing one of the built-in RNGs that ship with $\mathrm{R}$ is often a reasonable choice. The situation changes when it comes to parallel execution. Here, we need stream-aware random-number creation which is provided by both the older packages rlecuyer (Sevcikova et al., 2019) and rstream (Leydold, 2017) as well as the more-recent ones dqrng (Stubner, 2019) and sitmo (Balamuta and van den Berg, 2019).

Another technology worth mentioning is ArrayFire, provided for $\mathrm{R}$ by the (GitHub-only) package RcppArrayFire (Fukui and Stubner, 2019). It offers a C++ abstraction to highly-parallel code which can be executed in three different forms. The first is via multi-threading and OpenMP utilizing only the CPU. This provides a base case usable on most computers. But ArrayFire also provides wrappers to GPU code deploying either to CUDA and OpenCL, if available.

Finally, we mention package RhpcBLASctl (Nakano and Nakama, 2018) which provides a simple interface to querying and selecting the number of threads used for the OpenBLAS numerical analysis library but also for direct use of OpenMP (as it uses the same underlying environment variable).

\section{Machine-local Parallelism with Many Cores}

The previous section focused on a widely-available and widely-used technology. Due to its dependence on a $\mathrm{C}, \mathrm{C}++$ or Fortran compiler, it may be out of reach for some users. This section describes an alternative which can be seen as the opposite in terms of accessibility: using multiple cores on a single machine directly from $\mathrm{R}$.

Nowadays, machines with 'many cores' span the spectrum from servers to workstations and laptops. At the upper end, servers running the Linux operating system (or even Windows if one prefers or requires it) can have several dozen cores. The largest 'R5' instance on Amazon AWS is listed (in December 2019) as having 96 cores and 768 GB of memory. Base R, as shipped, can use this directly via the parallel package to deploy all of these 96 cores for (process-parallel) work. The parallel package was added to base R 2.14.0 (released in October 2011) after incorporating key components from 
the multicore and snow packages (already described by Schmidberger et al. (2009)). Both of these packages can be considered deprecated in favor of the parallel package.

In the simplest use case of package parallel, one replaces lapply () and mapply() with the corresponding "multicore" mclapply() and mcmapply() functions. The following example run an 'existence' proof requesting the process id of the running R process. It invokes mclapply () with argument $1: 4$ where a function of choice (here a simple request of the process id) is called in parallel on these elements. Because four elements and four cores (mc.cores $=4$ ) are used, the result of this call will be four unique process ids.

parallel::mclapply(1:4, function(i) Sys.getpid(), mc.cores $=4)$

A major advantage of the multicore processing in parallel due to forked parallel processing is that global variables in the main $\mathrm{R}$ session are inherited by the child processes. This means the developer does not have to spend efforts on identifying and exporting those to the parallel workers. However, one main disadvantage of forked parallel processes in combination with low-level multi-threaded processing is potential instability that may result in crashes and/or corrupt results. Because of this, multicore parallelization can only safely be used in cases where one has full control of all dependencies. If not, there is a risk that the implemented pipeline breaks because a previously single-threaded package dependency is updated to use multi-threaded processing. Another disadvantage of multicore processing is that it is only supported on Unix-like operating systems; on Microsoft Windows the type of forked processing needed is unsupported and therefore, if used, mclapply () and friends are designed to fall back to run in sequential mode.

An alternative to multicore processing is cluster processing, where parallelization takes place over a set of independent, non-interactive $\mathrm{R}$ processes running in the background and waiting for instructions from the main $\mathrm{R}$ session. The parallel package provides functions to create such clusters and $* \operatorname{apply}()$ like functions to invoke a function in parallel across a cluster, e.g. parLapply() and parMapply().

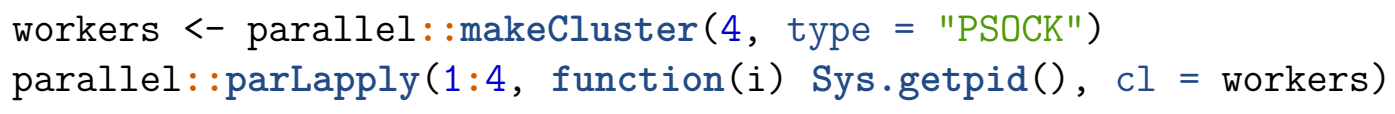

The advantages of this model is that it is supported on all operating systems, and that it also works with code that runs multi-threaded internally. The disadvantages are increased communication overhead, and that global variables have to be identified and explicitly exported to each worker in the cluster before processing. As discussed below, another advantage with cluster processing is that it supports also workers on external machines, possibly running in remote locations.

Similarly, other packages besides parallel can be deployed. We will discuss the future package (Bengtsson, 2019a) in more detail below, but one simple use case for it is to replace the parallel package. Packages snow (Tierney et al., 2018) (already described by Schmidberger et al. (2009)), snowfall (Knaus, 2015) (a once-popular "simpler" interface to snow) as well as foreach (Ooi et al., 2019b) with its companion packages doMC (Ooi et al., 2019a) and doParallel (Microsoft Corporation and Weston, 2019) also offer alternatives for such machine-local parallel tasks (as well as opportunities to launch multi-machine computations as discussed in the next section) but are seeing less active development than future.

\section{Local to Remote Parallelism with Message Passing}

The Message Passing Interface (MPI) standard, first described in Message Passing Interface Forum (1994), has long been a common tool in parallel and high-performance computing. The OpenMPI 
implementation of the MPI standard has now established itself as the reference implementation. It is being coordinated by an industry consortium just like the OpenMP standard discussed in the previous section. (Nowadays, the alternate MPICH implementation is less relevant, as is the competing PVM standard.)

In general, using MPI requires i) compiling and linking with a dedicated MPI library such as OpenMPI, and ii) running the binary in a setup with appropriate configuration. Given the message-passing nature, there (generally) needs to be other hosts ready to receive (and send) messages and to share the load (though for debugging and testing machine-local setups are of course also possible). At a minimum, authentication, permissioning and logging have to be provided making this a technology that is mostly provisioned by data centers. And, as above for OpenMP, programming with MPI at the $\mathrm{C}, \mathrm{C}++$ or Fortran level is not trivial. A quick scan of the CRAN package universe reveals eighteen packages including the mpi.h header file in their sources.

However, for parallel applications with $\mathrm{R}$, the higher-level Rmpi package (Yu, 2018) helps as it shields the user from some of the technicalities by offering simpler higher-level constructs. This was already described by Schmidberger et al. (2009) and has, by and large, not changed much. The packages snow (Tierney et al., 2018), snowfall (Knaus, 2015) and doMPI (Weston, 2017) are also still available and can be used for these tasks. As mentioned earlier, they are less actively developed than future (Bengtsson, 2019a) discussed below. When using snow, snowfall or doMPI, the underlying OpenMPI implementation can be used as the communications strategy in the cluster object, and functions such as parLapply() and parSapply() can be used to dispatch jobs across the cluster.

Some additional $\mathrm{R}$ packages of interest have emerged. Parallel computing workloads are often executed by schedulers and similar frameworks dealing with permissioning, quotas, logging, and other details. Among several of these schedulers and related tools, the Slurm workload manager has become one of the more popular choices. It offers a wide range of features, and is distributed under an open source license with optional commercial support. For use from $\mathrm{R}$, the rslurm package (Marchand et al., 2019) offers suitable integration. Its key functions comprise both an apply() variant named slurm_apply ( ) as well as single job launchers and job status query runners. Also of note is package batchtools (Lang et al., 2018) which operates in the same space and lets R user deploy tasks across (larger) computational clusters frequently used with MPI.

\section{Frameworks for Parallel Workloads and "Big Data"}

The previous three sections discussed what we may describe as "classic approaches" to parallel computing. These approaches were also already described in some form in Schmidberger et al. (2009). They also have one common element: all of them originate from, or are associated with, the more research-oriented or scientific side of computing that is commonly referred to as 'high-performance computing' or 'scientific computing'.

Focusing on the other end of the spectrum, this section now turns towards the 'internet-scale' age of 'Big Data'. We will look at frameworks for analysing large data sets via Hadoop and Spark, two technologies that have been dominating this area since the report by Schmidberger et al. (2009) (which did not foresee them) came out. We will then turn to containers for development and deployment which can also be used for parallel computing.

Hadoop and Spark. The Hadoop File System (Shvachko et al., 2010), frequently referred to as just 'Hadoop', started as a re-implementation of two key Google technologies: MapReduce, and the Google File System. Its key attraction is the "unlimited" scope of data it can process as a decentralized federation of machines can be assembled. Hadoop, and later Spark, clusters can be used "on premises" (i.e. 
in local data centers) as well as in cloud-based deployments. An entire eco-system of related services such as the Hadoop Distributed File System and the Yarn resource manager / scheduler emerged over time. All of these components eventually became part of the larger Apache Project. Hadoop may now be considered well-established and is certainly widely used, especially in industry, but is generally no longer considered to be cutting edge.

Spark (Zaharia et al., 2010, 2016), also an Apache Project, originated at the University of California, Berkeley. It offers resilient distributed data structures organized as data.frame objects, making it suitable for very large data sets. Access from $\mathrm{R}$ is possible via several projects, notably SparkR (Venkataraman et al., 2019) and sparklyr (Luraschi et al., 2019). Here the first package goes back to the Apache Spark project itself, whereas the second package is a RStudio product and well integrated into their offerings. This combines the strength of Spark and its ability to access large distributed data sets with the familiar R front-ends and operations, especially the dplyr package (Wickham et al., 2019) for which sparklyr can act as a back-end and connection to Spark.

At the time of writing, Spark can be considered as the dominant "big data" platform in industry. Essentially all data-storage and processing back-ends as well as analytics libraries connect to it, and can compute results within Spark-based workflows. Spark, just like Hadoop, has a reasonably strong connection to the Java ecosystem, and many integrations utilize the Java Virtual Machine abstraction. It is however also a 'wire protocol' so non-JVM approaches can be used with it as well.

Docker and Kubernetes. Another important and recent trend not anticipated in Schmidberger et al. (2009) is containerization. Probably best-known, and sometimes taken as synonymous, is Docker (Merkel, 2014). At its core, Docker relies on Linux-specific resource management and isolation capabilities such as kernel namespaces and cgroups to allow independent entities (the "containers") to run within a single Linux host instance while avoiding the heavier overhead of full virtual machines. Docker can be used on other operating systems by providing a minimal layer of code that provides these calls; in the simplest cases a virtual machine may be used. Docker is one of several technologies using these features, but has become 'the public face' of container use eclipsing related approaches such as the "Linux Containers" technology LXC (Wikipedia Contributors, 2019b), or the Singularity approach (Kurtzer et al., 2017) coming from the high-performance computing side.

Within the R world, the Rocker Project, described by Boettiger and Eddelbuettel (2017), offers a wide variety of R-based containers. These range from smaller and lighter-weight containers providing just R (as well as development and test version of R) to fuller-featured containers with RStudio Server, Shiny Server, or some larger collections of packages, for example for geo-spatial computing. Another popular series of containers 'snapshots' to the R releases ensuring reproducibility for various "points in time". Containers from the Rocker Project also form the building blocks for a large number of products utilizing their standardized packaging and provisioning of the R system and packages.

Containerization, due to some key architectural and implementation aspects, generally wraps a single process. This is a key feature which makes it easy to "wrap" a single program or application along with all its required libraries or components, and a clear key to its wide adoption and success. Yet another common use case may involve the need to have multiple programs operate in a coordinated manner. Such "orchestration" proved to be a very natural second step for Docker, and multiple approaches have been tried.

Among these, the Kubernetes system (Wikipedia Contributors, 2019a) for container orchestration originally started by Google (and now developed by the Cloud Native Computing Foundation) has become the dominant entry. Kubernetes is generally supported by cloud-computing providers as a basic platform or infrastructure building block. It is frequently combined with Spark, which we discussed 
in the previous section, to provision "big data" computing at scale.

\section{Application Focus: future}

The future package by Bengtsson (2019a) provides a very attractive and cohesive application layer for several topics discussed above. It builds upon the concepts of futures (Hewitt and Baker, 1977) and promises (Friedman and Wise, 1978; Hibbard, 1976). With a single unified application-programming interface (API), it can replace simple uses cases such as the mclapply() example above. Yet at the same time it can also scale to multi-machine or multi-host parallel computing using a variety of parallel computing back-ends.

One key element is an underlying abstraction from asynchronous computing: future allows to "launch" a compute task and access / retrieve its result "when it is ready" (or block until it is). Consider a base R expression such as a <- expr where the expression part evaluating expr will typically involve some computation. Using the API of the future package, we can write $f<-$ future (expr) which "hands" the expression to the future package, and a (later, or immediate) $v<-$ value $(f)$. The state of this future value is either resolved, or unresolved in which case further execution may block until it is resolved.

As an illustration, consider the following example borrowed from the overview vignette. Simply by switching the plan () between sequential, multi-process and multi-machine, the well-known (and time-consuming) Mandelbrot Set illustration can be rendered in three different ways at three different speeds.

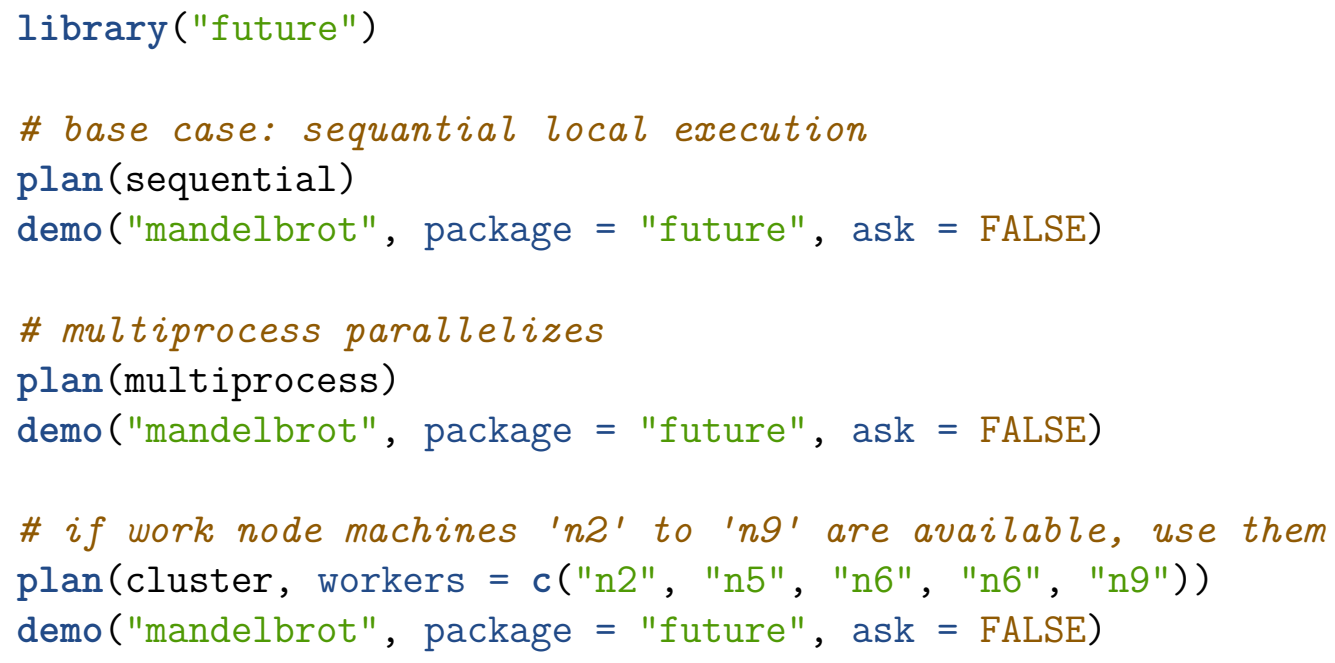

The future package is complemented by several additional packages. The future.apply package (Bengtsson, 2019b) offers variants of R's *apply functions, similar to the solutions discussed above but also addressing load balancing aspects, that work with the different back-ends supported by future. Back-ends are generally selected by issueing a plan() statement with an appropriate processing plan. This covers multi-process, cluster, as well as support for computing-center scale via slurm, batchtools and more. In general, each specific back-end is provided by an additional (custom) package.

Future offers a very consistent API. This facilitates adapting deployment to different usage scenarios or backends. In many cases, users will only have to change the plan() statement which serves as the ex ante declaration of the deployment. Further, several detailed vignettes document usage of the future package, its related packages, and its various options. 


\section{Summary}

Parallel computing is a tool which can help in the context of large and demanding analysis tasks, especially when these are seen to be "data-parallel" without interdependence or linkage between the tasks. For the R user and analyst, parallel computing can take many forms. This survey delineates the various possibilities from the inside out. Starting with from 'CPU-local' and compiler-dependendent methods like OpenMP and Intel TBB, we looked at the machine-local multiprocessing approaches, before discussing message-passing parallelism as offered by OpenMPI. (Relatively) newer approaches centered on "big data" such as Spark, Docker and Kubernetes rounded out the review along with an application-level highlight offered by the future package.

It is our hope that this survey proves to be of use for analysts and researchers wishing to analyse and model with $\mathrm{R}$ in a parallel manner.

\section{Acknowledgements}

This paper has benefited greatly from conversations with James Balamuta and Henrik Bengtsson whose insightful suggestions are truly appreciated. Further comments by the David Scott, the editor, and two anonymous referees were also very helpful and are gratefully acknowledged.

\section{References}

Abadi M, Agarwal A, Barham P, Brevdo E, Chen Z, Citro C, Corrado GS, Davis A, Dean J, Devin M, Ghemawat S, Goodfellow I, Harp A, Irving G, Isard M, Jia Y, Jozefowicz R, Kaiser L, Kudlur M, Levenberg J, Mané D, Monga R, Moore S, Murray D, Olah C, Schuster M, Shlens J, Steiner B, Sutskever I, Talwar K, Tucker P, Vanhoucke V, Vasudevan V, Viégas F, Vinyals O, Warden P, Wattenberg M, Wicke M, Yu Y, Zheng X (2015). "TensorFlow: Large-Scale Machine Learning on Heterogeneous Systems." Software available from tensorflow.org, URL https://www.tensorflow.org/.

Allaire J, Francois R, Ushey K, Vandenbrouck G, Geelnard M, Intel (2019). RcppParallel: Parallel Programming Tools for 'Rcpp'. R package version 4.4.4, URL https://CRAN.R-project.org/package=RcppParallel.

Balamuta J, van den Berg T (2019). sitmo: Parallel Pseudo Random Number Generator (PPRNG) 'sitmo' Header Files. R package version 2.0.1, URL https://CRAN.R-project.org/package=sitmo.

Bengtsson H (2019a). future: Unified Parallel and Distributed Processing in $R$ for Everyone. R package version 1.15.1, URL https://CRAN.R-project.org/package=future.

Bengtsson H (2019b). future.apply: Apply Function to Elements in Parallel using Futures. $\mathrm{R}$ package version 1.3.0, URL https://CRAN.R-project.org/package=future.apply.

Boettiger C, Eddelbuettel D (2017). "An Introduction to Rocker: Docker Containers for R." The $R$ Journal, 9(2), 527-536. URL https://journal.r-project.org/archive/2017/RJ-2017-065/index.html.

Chen T, He T, Benesty M, Khotilovich V, Tang Y, Cho H, Chen K, Mitchell R, Cano I, Zhou T, Li M, Xie J, Lin M, Geng Y, Li Y (2019). xgboost: Extreme Gradient Boosting. R package version 0.90.0.2, URL https://CRAN.R-project.org/package=xgboost.

Clayden J, Modat M, Presles B, Anthopoulis T, Daga P (2019). RNiftyReg: Image Registration Using the 'NiftyReg' Library. R package version 2.6.7, URL https://CRAN.R-project.org/package=RNiftyReg.

Dagum L, Menon R (1998). "OpenMP: An Industry-Standard API for Shared-Memory Programming." IEEE Comput. Sci. Eng., 5(1), 46-55. ISSN 1070-9924. doi:10.1109/99.660313. URL https://doi.org/10.1109/99.660313.

Determan Jr C (2019). RViennaCL: 'ViennaCL' C++ Header Files. R package version 1.7.1.8, URL https://CRAN.R-project.org/package=RViennaCL.

Dowle M, Srinivasan A (2019). data.table: Extension of 'data.frame'. R package version 1.12.6, URL https://CRAN.R-project.org/package=data.table.

Eddelbuettel D, Balamuta JJ (2018). "Extending R with C++: A Brief Introduction to Rcpp." The American Statistician, 72(1), 28-36. doi:10.1080/00031305.2017.1375990. https://doi.org/10.1080/00031305.2017.1375990, URL https://doi.org/10.1080/00031305.2017.1375990.

Eddelbuettel D, Francois R, Allaire J, Ushey K, Kou Q, Russell N, Bates D, Chambers J (2019a). Rcpp: Seamless R and C++ Integration. Http://www.rcpp.org, http://dirk.eddelbuettel.com/code/rcpp.html, https://github.com/RcppCore/Rcpp.

Eddelbuettel D, Francois R, Bates D, Ni B (2019b). RcppArmadillo: 'Rcpp' Integration for the 'Armadillo' Templated Linear Algebra Library. R package version 0.9.800.3.0, URL https://CRAN.R-project.org/package=RcppArmadillo.

Friedman DP, Wise DS (1978). "Aspects of Applicative Programming for Parallel Processing." IEEE Transactions on Computers, C-27(4), 289-296. doi:10.1109/tc.1978.1675100.

Fukui K, Stubner R (2019). RcppArrayFire: Rcpp integration for ArrayFire. GitHub-based R package version 0.1.0, URL https://github.com/daqana/rcpparrayfire.

Goodrich B, Gelman A, Carpenter B, Hoffman M, Lee D, Betancourt M, Brubaker M, Guo J, Li P, Riddell A, Inacio M, Morris M, Arnold J, Goedman R, Lau B, Trangucci R, Gabry J, Kucukelbir A, Grant R, Tran D, Malecki M, Gao Y (2019). StanHeaders: C++ Header Files for Stan. R package version 2.19.0, URL https://CRAN.R-project.org/package=StanHeaders.

Hewitt C, Baker HG (1977). "Laws for Communicating Parallel Processes." In IFIP Congress, pp. 987-992. URL https://dblp.uni-trier.de/db/conf/ifip/ifip1977.html\#HewittB77.

Hibbard P (1976). "Parallel Processing Facilities." In SA Schuman (ed.), New Directions in Algorithmic Languages. IRIA.

Knaus J (2015). snowfall: Easier cluster computing (based on snow). R package version 1.84-6.1, URL https://CRAN.R-project.org/package=snowfall. 
Kurtzer GM, Sochat V, Bauer MW (2017). "Singularity: Scientific containers for mobility of compute." PLOS ONE, 12(5), e0177459. ISSN 1932-6203. doi:10.1371/journal . pone.0177459. URL http://journals.plos.org/plosone/article?id=10.1371/journal.pone.0177459.

Lang M, Bischl B, Surmann D (2018). batchtools: Tools for Computation on Batch Systems. R package version 0.9.11, URL https://CRAN.R-project.org/package=batchtools.

Leydold J (2017). rstream: Streams of Random Number. R package version 1.3.5, URL https://CRAN.R-project.org/package=rstream.

Luraschi J, Kuo K, Ushey K, Allaire J, Macedo S, RStudio, The Apache Software Foundation (2019). sparklyr: R Interface to Apache Spark. R package version 1.0.5, URL https://CRAN.R-project.org/package=sparklyr.

Marchand P, Carroll I, Smorul M, Blake R, Read Q (2019). rslurm: Submit R Calculations to a 'Slurm' Cluster. R package version 0.5.0, URL https://CRAN.R-project.org/package=rslurm.

Merkel D (2014). "Docker: Lightweight Linux Containers for Consistent Development and Deployment.” Linux Journal, 2014(239). ISSN 1075-3583. URL http://dl.acm.org/citation.cfm?id=2600239.2600241.

Mersmann O (2019). microbenchmark: Accurate Timing Functions. R package version 1.4-7, URL https://CRAN.R-project.org/package=microbenchmark.

Message Passing Interface Forum (1994). "MPI: A Message-Passing Interface Standard." Technical report, Knoxville, TN, USA.

Microsoft Corporation, Weston S (2019). doParallel: Foreach Parallel Adaptor for the 'parallel' Package. R package version 1.0.15, URL https://CRAN.R-project.org/package=doParallel.

Morandat F, Hill B, Osvald L, Vitek J (2012). "Evaluating the Design of the R Language." In J Noble (ed.), ECOOP 2012 - Object-Oriented Programming, pp. 104-131. Springer Berlin Heidelberg, Berlin, Heidelberg. ISBN 978-3-642-31057-7.

Nakano J, Nakama E (2018). RhpcBLASctl: Control the Number of Threads on 'BLAS'. R package version 0.18-205, URL https://CRAN.R-project.org/package=RhpcBLASctl.

Ooi H, Microsoft, Weston S (2019a). doMC: Foreach Parallel Adaptor for 'parallel'. R package version 1.3.6, URL https://CRAN.R-project.org/package=doMC.

Ooi H, Microsoft, Weston S (2019b). foreach: Provides Foreach Looping Construct. R package version 1.4.7, URL https://CRAN.R-project.org/package=foreach.

Paszke A, Gross S, Massa F, Lerer A, Bradbury J, Chanan G, Killeen T, Lin Z, Gimelshein N, Antiga L, Desmaison A, Kopf A, Yang E, DeVito Z, Raison M, Tejani A, Chilamkurthy S, Steiner B, Fang L, Bai J, Chintala S (2019). "PyTorch: An Imperative Style, High-Performance Deep Learning Library." In H Wallach, H Larochelle, A Beygelzimer, F d'Alché-Buc, E Fox, R Garnett (eds.), Advances in Neural Information Processing Systems 32, pp. 8024-8035. Curran Associates, Inc. URL http://papers.neurips.cc/paper/9015-pytorch-an-imperative-style-high-performance-deep-learning-library.pdf.

R Core Team (2019). R: A Language and Environment for Statistical Computing. R Foundation for Statistical Computing, Vienna, Austria. URL https://www.R-project.org/.

Roehl T, Treibig J, Hager G, Wellein G (2014). "Overhead Analysis of Performance Counter Measurements." In 43rd International Conference on Parallel Processing Workshops (ICCPW), pp. 176-185. ISSN 1530-2016. doi: 10.1109/ICPPW. 2014. 34.

Sartore L (2019). spMC: Continuous-Lag Spatial Markov Chains. R package version 0.3.10, URL https://CRAN.R-project.org/package=spMC.

Schmidberger M, Morgan M, Eddelbuettel D, Yu H, Tierney L, Mansmann U (2009). "State of the Art in Parallel Computing with R." Journal of Statistical Software, Articles, 31(1), 1-27. ISSN 1548-7660. doi:10.18637/jss.v031.i01. URL https://www.jstatsoft.org/v031/i01.

Sevcikova H, Rossini T, L'Ecuyer P (2019). rlecuyer: $R$ Interface to $R N G$ with Multiple Streams. $R$ package version 0.3-5, URL https://CRAN.R-project.org/package=rlecuyer.

Shvachko K, Kuang H, Radia S, Chansler R (2010). "The Hadoop Distributed File System." In Proceedings of the 2010 IEEE 26 th Symposium on Mass Storage Systems and Technologies (MSST), MSST '10, pp. 1-10. IEEE Computer Society, Washington, DC, USA. ISBN 978-1-4244-7152-2. doi:10.1109/MSST . 2010.5496972. URL http://dx.doi.org/10.1109/MSST.2010.5496972.

Stubner R (2019). dqrng: Fast Pseudo Random Number Generators. R package version 0.2.1, URL https://CRAN.R-project.org/package=dqrng.

Tierney L, Rossini AJ, Li N, Sevcikova H (2018). snow: Simple Network of Workstations. R package version 0.4-3, URL https://CRAN.R-project.org/package=snow.

Treibig J, Hager G, Wellein G (2010). "LIKWID: A lightweight performance-oriented tool suite for x86 multicore environments." In Proceedings of PSTI2010, the First International Workshop on Parallel Software Tools and Tool Infrastructures. San Diego CA.

Venkataraman S, Meng X, Cheung F, The Apache Software Foundation (2019). SparkR: R Front End for 'Apache Spark'. R package version 2.4.4, URL https://CRAN.R-project.org/package=SparkR.

Weston S (2017). doMPI: Foreach Parallel Adaptor for Rmpi Package. R package version 0.2.2, URL https://CRAN.R-project.org/package=doMPI.

Wickham H, François R, Henry L, Müller K (2019). dplyr: A Grammar of Data Manipulation. R package version 0.8.3, URL https://CRAN.R-project.org/package=dplyr.

Wikipedia Contributors (2019a). "Kubernets." Online; last accessed 23-December-2019, URL https://en.wikipedia.org/wiki/Kubernetes.

Wikipedia Contributors (2019b). "LXC." Online; last accessed 23-December-2019, URL https://en.wikipedia.org/wiki/LXC.

Wikipedia Contributors (2019c). "SIMD." Online; accessed 2-December-2019, URL https://en.wikipedia.org/wiki/SIMD.

Yu H (2018). Rmpi: Interface (Wrapper) to MPI (Message-Passing Interface). R package version 0.6-9, URL https://CRAN.R-project.org/package=Rmpi.

Zaharia M, Chowdhury M, Franklin MJ, Shenker S, Stoica I (2010). "Spark: Cluster Computing with Working Sets." In Proceedings of the 2Nd USENIX Conference on Hot Topics in Cloud Computing, HotCloud'10, pp. 10-10. USENIX Association, Berkeley, CA, USA. URL http://dl.acm.org/citation.cfm?id=1863103.1863113.

Zaharia M, Xin RS, Wendell P, Das T, Armbrust M, Dave A, Meng X, Rosen J, Venkataraman S, Franklin MJ, et al. (2016). "Apache spark: a unified engine for big data processing." Communications of the ACM, 59(11), 56-65. 